\title{
Incidence and predictors of sexually transmitted infections among adult HIV-positive patients receiving antiretroviral therapy at Themba Lethu HIV clinic in Johannesburg, South Africa
}

\author{
D Onoya, ${ }^{1} \mathrm{PhD}$; P Manjengwa, ${ }^{1} \mathrm{MPH} ; \mathrm{C}$ Nattey, ${ }^{1} \mathrm{MSc} ;$ S Kgowedi, ${ }^{1} \mathrm{BSc} ;$ M Mbele, ${ }^{2} \mathrm{MB}$ ChB; K Shumba, ${ }^{3} \mathrm{MB}$ ChB; $\mathbf{M}$ P Fox ${ }^{1,4,5}$ DSc \\ ${ }^{1}$ Health Economics and Epidemiology Research Office, Department of Internal Medicine, School of Clinical Medicine, Faculty of Health Sciences, \\ University of the Witwatersrand, Johannesburg, South Africa \\ ${ }^{2}$ Right to Care, Johannesburg, South Africa \\ ${ }^{3}$ School of Public Health, Faculty of Health Sciences, University of the Witwatersrand, Johannesburg, South Africa \\ ${ }^{4}$ Department of Global Health, Boston University School of Public Health, Boston, Mass, USA \\ ${ }^{5}$ Department of Epidemiology, Boston University School of Public Health, Boston, Mass, USA
}

Corresponding author: D Onoya (donoya@heroza.org)

\begin{abstract}
Background. Sexually transmitted infections (STIs) among people living with HIV/AIDS can facilitate the spread of HIV.
Objectives. To estimate STI incidence and identify predictors of STI acquisition among HIV-positive patients during their first 24 months of antiretroviral therapy (ART) in Johannesburg, South Africa.

Methods. We conducted a cohort study using prospectively collected routine data on patients who initiated ART between January 2004 and January 2015 at the Themba Lethu HIV clinic in Johannesburg. Kaplan-Meier analysis was used to estimate STI incidence rates (based on evidence of laboratory diagnosis and STI syndromic treatment prescription records). STI predictors were identified using Cox regression analysis.

Results. Among 26762 adult patients on ART, there were 1906 (7.1\%) cases of STI (incidence 4.8/100 person-years; 95\% confidence interval (CI) 4.7 - 5.1). Non-pregnant women were $60 \%$ more likely than men to be diagnosed with an STI (adjusted hazard ratio (aHR) 1.6; 95\% CI 1.4 - 1.8). The risk of STI decreased with increasing baseline CD4 count (aHR 0.8, 0.5 and 0.4 for CD4 counts $101-200$, $201-350$ and $>350$ cells $/ \mu \mathrm{L}$, respectively, compared with CD4 count $<100$ cells $/ \mu \mathrm{L}$ ). Patients with advanced baseline World Health Organization (WHO) clinical stages had a higher risk (aHR 1.6 for WHO stage 4; 95\% CI 1.3 - 1.9) compared with those with WHO stage 1. However, there was a $20 \%$ increase in the risk of STI among obese patients compared with underweight patients (aHR 1.3; 95\% CI 1.0 - 1.7). Over $80 \%$ of obese patients diagnosed with an STI had a CD 4 count $<200$ cells $/ \mu \mathrm{L}$.

Conclusions. STIs are common in HIV-infected patients who are receiving ART. While both ART and the syndromic management of STIs are high-impact interventions for controlling the spread of HIV, closer monitoring of STI occurrences is warranted, particularly among immunologically vulnerable patients.
\end{abstract}

S Afr Med J 2021;111(1):80-86. https://doi.org/10.7196/SAMJ.2021.v111i1.14672

Sexually transmitted infections (STIs) constitute important public health concerns in developing countries, where they account for $17 \%$ of illness-related economic losses. ${ }^{[1]}$ In South Africa (SA), a Spectrum model estimation exercise in 2017 reported Chlamydia trachomatis (CT) prevalence at $14.7 \%$ for men and $6.0 \%$ for women, Neisseria gonorrhoeae (NG) prevalence at 6.6\% for men and 3.5\% for women, and active syphilis prevalence at $0.5 \%$ for men and $0.97 \%$ for women. ${ }^{[2]}$ The 2015 SA antenatal care (ANC) survey reported syphilis prevalence of $2.0 \%$ among pregnant women attending public sector facilities, similar to levels observed in 2007 $2008 .{ }^{[3]}$ In a 2013 study among adults in Johannesburg, 20.4\% of all participants self-reported an STI episode (19.9\% among HIVinfected participants). ${ }^{[4]}$

Untreated STIs can lead to long-term sequelae of infection. In adults, such manifestations occur in the reproductive system (genital ulceration, infertility, pelvic inflammatory disease), neurological system or cardiovascular system, and in infants, blindness or severe neurological disability is common. ${ }^{[5-7]}$ The prevention and early treatment of STIs are particularly crucial for SA because of the country's high HIV burden. SA was estimated to have 7.9 million individuals living with HIV in 2017. ${ }^{[8]}$ Considering the high probability of HIV transmission when either sexual partner has an STI, ${ }^{[9,10]}$ a high STI incidence among individuals living with HIV has considerable implications for containing the spread of HIV in general.

Several studies in developing countries have shown that treating STIs among HIV-infected patients significantly reduces the risk of transmission by reducing levels of HIV in genital secretions. ${ }^{[2,9]}$ In 1995, SA adopted the syndromic approach to STI management, which entails the rapid treatment of STI cases identified by symptom screening rather than specific aetiology ${ }^{[11]}$ Slight increases in STI prevalence were observed between 1990 and 2005 for NG 
(52.5\% to $54.4 \%$ among women and $30.7 \%$ to $31.7 \%$ among men), while decreases were noted for trichomoniasis $30.9 \%$ to $24.2 \%$ among women and $5.9 \%$ to $4.0 \%$ among men), CT (11.5\% to $10.1 \%$ among women and $8.7 \%$ to $7.1 \%$ among men) and syphilis ( $7.7 \%$ to $1.9 \%$ among women and $7.4 \%$ to $2.2 \%$ among men). Genital herpes simplex virus (HSV-2) prevalence appears to have increased slightly over time $(52.5 \%$ to $54.4 \%$ among women and $30.7 \%$ to $31.7 \%$ among men). ${ }^{[2,9]}$ There is some evidence to suggest that genital ulcers are more likely to occur among HSV-2-infected individuals who are HIV-immunocompromised, and as the HIV epidemic matures, the contribution of HSV-2 to HIV transmission also increases. ${ }^{[12]}$ However, the proportion of new HIV infections in adults that were attributable to curable STIs also declined, from $39 \%$ in 1990 to $14 \%$ in $2010 .^{[2,9]}$

Despite its widespread benefits, syndromic STI management limits opportunities for diagnosis of asymptomatic infections and has been associated with poor reporting of STI cases. ${ }^{[11,13]}$ Many of the modelled STI prevalence estimates in SA rely heavily on data from ANC surveys, research studies and clinical trials. There is limited information on STI incidence rates across both HIVinfected and uninfected populations, and even fewer data for HIV-infected individuals who are receiving antiretroviral therapy (ART). ${ }^{[2,14]}$

\section{Objectives}

To estimate the incidence of STIs among HIV-positive adults receiving care at the Themba Lethu HIV clinic in Johannesburg, SA.

\section{Methods \\ Study population}

We conducted a cohort study using prospectively collected data on adult HIV-positive (age $\geq 18$ years) patients accessing standard firstline ART ${ }^{[15]}$ at the Themba Lethu HIV clinic in Johannesburg from January 2004 to January 2015. At the time of data collection, clinical data at Themba Lethu were routinely captured and stored in an electronic patient management system (TherapyEdge-HIV; Devex, USA). SA follows the syndromic approach for managing STIs, where patients are given a combination regimen based on the outcomes of a symptom screen during a medical visit. An STI symptom screen is recommended for all Themba Lethu ART patients every 3 months. Study participants were followed up via medical record review over 24 months after ART initiation to identify incident STIs. Incident STIs were determined from laboratory data, clinical notes and treatment prescription records, as defined in Table 1. ${ }^{[16]}$ As such, patients prescribed regimens consisting of recommended drugs for syndromic STI management on the clinic visits were also identified as incident STI cases in consultation with a prescribing clinician at the HIV clinic.

Demographic variables collected at the time of ART initiation (baseline) include age, gender, highest level of education (categorised as no education, primary school and secondary school), history of smoking (yes/no), history of alcohol use (yes/no) and employment status (employed/unemployed). Baseline clinical data include CD4 count, World Health Organization (WHO) clinical stage and body mass index (BMI). The primary outcome variable was the occurrence of an incident STI over 24 months after first-line ART initiation. Person-time accrued from the date of ART initiation to the first occurrence of an STI or the last time seen at the clinic (for patients who were lost to follow-up or transferred out, or died), or to the end of the observation period ( 24 months). Data were fully anonymised before statistical analysis. The study was approved by the Human Research Ethics Committee (Medical) of the University of the Witwatersrand (ref. no. M170564).

\section{Statistical analysis}

Continuous variables were described using medians and interquartile ranges (IQRs). Categorical variables were described using percentages. Kaplan-Meier analyses were conducted to determine STI incidence rates. Predictors of incident STIs among treated HIV-positive patients were modelled using Cox proportional hazards modelling. As our goal was to identify predictors and not assess causation, variables with a $p$-value $<0.1$ in crude analyses were included in a multivariate model. Schoenfeld residuals were used to test the assumption of proportional hazards. Variables were excluded from the model when the inclusion of the interaction term did not resolve the proportional hazards assumption violation, in which case the model was stratified.

\section{Results}

\section{Cohort description and baseline characteristics}

Table 2 shows the distribution of baseline demographic and clinical characteristics of the 26762 eligible patients. The overall median (IQR) age of the participants was 36.7 (31.0 - 43.4) years. Overall, $61.4 \%$ of participants were female. Female subjects were more likely than males to be $\leq 30$ years old at ART initiation (29.2\% v. $15.0 \%)$. Overall, $49.0 \%$ of the study participants were unemployed and $79.1 \%$ had a secondary-school level of education at ART initiation. Furthermore, a higher proportion of males than females reported a baseline history of alcohol consumption $(24.8 \% \mathrm{v} .5 .4 \%)$ or a history of smoking ( $18.6 \%$ v. $5.7 \%)$.

Overall, $6.7 \%$ of the study sample had a history of tuberculosis at the time of ART initiation.

\section{STIs in HIV-positive adults on ART}

A total of 1906 (7.1\%) STI cases were identified. Of these, 708 were identified using clinical notes with prescribed medicines, and the remaining 1198 were determined using the prescription information. Table 3 shows the proportions of STIs using the clinical notes with prescribed medication and prescription information (syndromic management). Of the 708 patients, $16.3 \%$ had anogenital warts, $13.7 \%$ had HSV-2 and only $2 \%$ had chancroid. The regimen-based STI cases were mostly prescribed metronidazole + ciprofloxacin $(32.8 \%)$, metronidazole + ciprofloxacin + doxycycline $(27.5 \%)$ or metronidazole + doxycycline $(19.3 \%)$ (Table 4$)$.

The incidence of STIs was 4.9/100 person-years (py) (95\% confidence interval (CI) 4.7 - 5.1). Among patients who had an incident STI on ART, 126 (6.6\%) had two or more episodes during the period of observation. Only $213(11.2 \%)$ of STI cases had HIV viral load data up to 6 months before or after the STI episode, and $74.2 \%$ of these were virally suppressed. The median (IQR) time to the first STI episode was $3.1(0.7-9.9)$ months from the time of ART initiation.

Table 5 presents predictors of incident STIs among HIV-positive patients on ART. Women who were not pregnant at ART initiation were $60 \%$ more likely than men to have an incident STI (adjusted hazard ratio (aHR) 1.6; 95\% CI 1.4 - 1.8) (Fig. 1). The risk of STI decreased with increasing age (aHR 0.6 for patients aged 41 50 years compared with $\leq 30$ years; $95 \%$ CI $0.5-0.7)$. Patients with a low baseline CD 4 count $(<100$ cells $/ \mu \mathrm{L})$ were at increased risk of incident STIs, with the risk decreasing with increasing baseline CD4 count (aHR 0.8, 0.5 and 0.4 for CD4 counts $101-200,201-350$ and $>350$ cells $/ \mu \mathrm{L}$, respectively, compared with CD 4 count $<100$ cells $/ \mu \mathrm{L}$ ). 


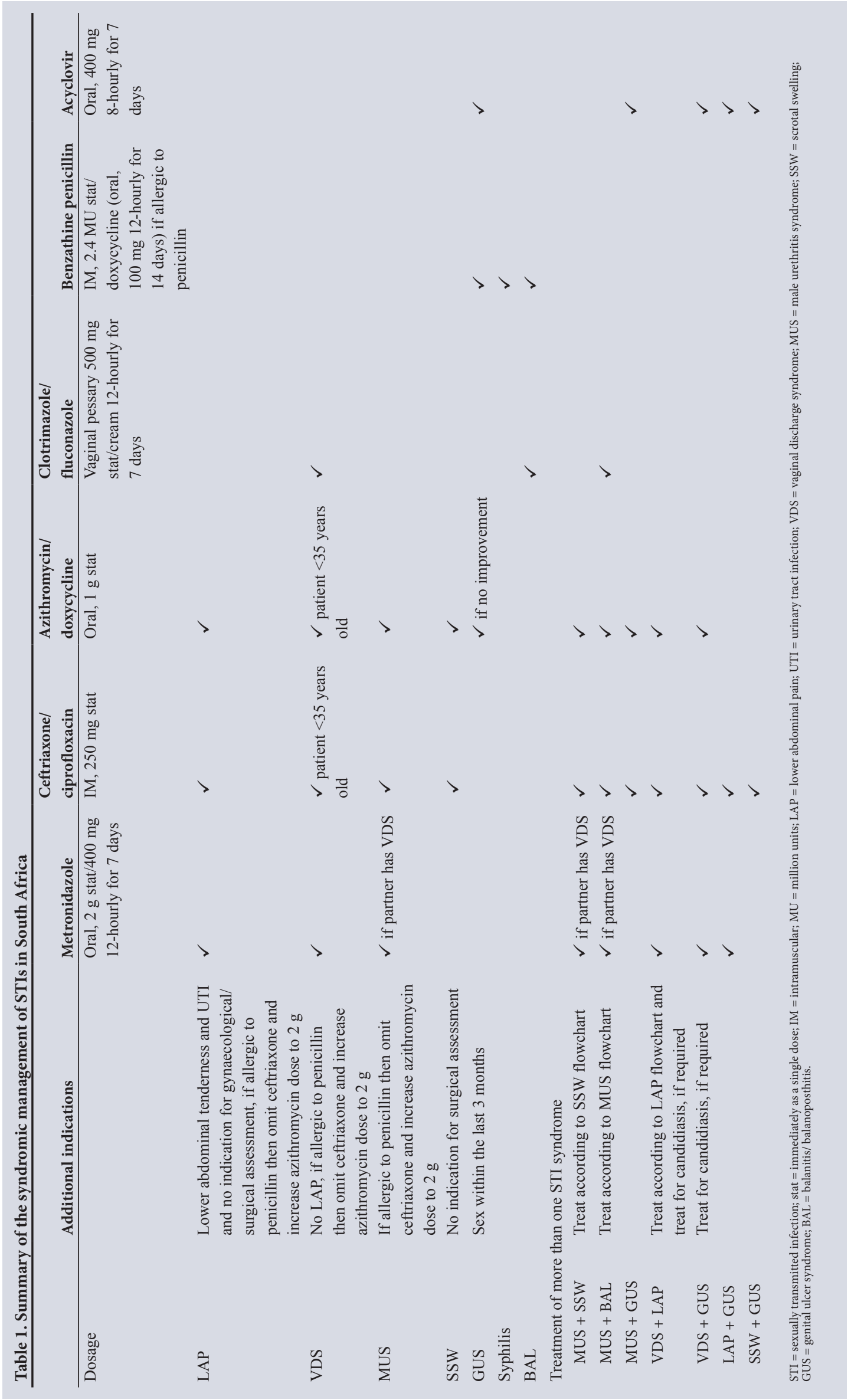


Table 2. Demographic characteristics of patients on ART in a public hospital in Johannesburg, South Africa, by sex and pregnancy status at ART start (among females)

\begin{tabular}{|c|c|c|c|c|}
\hline & $\begin{array}{l}\text { Female, pregnant at ART } \\
\text { start }(N=280), n(\%)\end{array}$ & $\begin{array}{l}\text { Female, not pregnant at ART } \\
\text { start }(N=16150), n(\%)\end{array}$ & $\begin{array}{l}\text { Male }(N=10332), \\
n(\%)\end{array}$ & $\begin{array}{l}\text { Total }(N=26762), \\
n(\%)\end{array}$ \\
\hline \multicolumn{5}{|l|}{ Age (years) at ART start } \\
\hline$\leq 30$ & $148(52.9)$ & $4649(28.8)$ & $1550(15.0)$ & $6347(23.7)$ \\
\hline $31-40$ & $130(46.4)$ & $6652(29.3)$ & $4844(46.9)$ & $11629(43.4)$ \\
\hline $41-50$ & $1(0.4)$ & $3489(39.4)$ & $2790(27.0)$ & $6280(23.5)$ \\
\hline$\geq 51$ & $1(0.4)$ & $4409(27.1)$ & $1148(11.1)$ & $2509(9.4)$ \\
\hline \multicolumn{5}{|c|}{ Employment status at ART start } \\
\hline No & $154(55.6)$ & $4374(43.4)$ & $4393(43.2)$ & $12779(49.0)$ \\
\hline Yes & $123(44.4)$ & $5693(56.6)$ & $5766(56.8)$ & $13325(51.0)$ \\
\hline \multicolumn{5}{|c|}{ Education level at ART start } \\
\hline No education & $8(3.5)$ & $720(5.5)$ & $514(6.1)$ & $1242(5.7)$ \\
\hline Primary education & $21(9.3)$ & $1866(14.2)$ & $1406(16.8)$ & $3293(15.2)$ \\
\hline Secondary education & $197(87.2)$ & $10537(80.3)$ & $6448(77.1)$ & $17182(79.1)$ \\
\hline \multicolumn{5}{|c|}{$\mathrm{CD} 4($ cells $/ \mu \mathrm{L})$ at $\mathrm{ART}$ start } \\
\hline $0-100$ & $70(29.9)$ & $5858(44.1)$ & $4571(52.5)$ & $10499(47.2)$ \\
\hline $101-200$ & $95(40.6)$ & $4284(32.2)$ & $2518(28.9)$ & $6897(31.0)$ \\
\hline $201-350$ & $56(23.1)$ & $2437(18.3)$ & $1319(15.1)$ & $3810(17.1)$ \\
\hline$>350$ & $15(6.4)$ & $715(5.4)$ & $302(3.5)$ & $1032(4.6)$ \\
\hline \multicolumn{5}{|l|}{ BMI $\left(\mathrm{kg} / \mathrm{m}^{2}\right)$ at ART start } \\
\hline$<18.5$ & $15(6.7)$ & $1898(15.1)$ & $1937(24.1)$ & $3850(18.7)$ \\
\hline $18.5-24.9$ & $113(50.4)$ & $6245(50.8)$ & $5075(63.1)$ & $11433(55.6)$ \\
\hline $25-29.9$ & $66(29.5)$ & $2652(21.6)$ & $844(10.4)$ & $3562(17.3)$ \\
\hline$\geq 30$ & $30(13.4)$ & $1506(12.2)$ & $183(2.3)$ & $1719(8.4)$ \\
\hline \multicolumn{5}{|l|}{ WHO stage at ART start } \\
\hline 1 & $218(77.9)$ & $10921(67.6)$ & $6362(61.6)$ & $17501(65.4)$ \\
\hline 2 & $2(0.7)$ & $201(1.2)$ & $98(0.9)$ & $301(1.1)$ \\
\hline 3 & $49(17.5)$ & $4049(25.1)$ & $3042(29.3)$ & $7140(26.7)$ \\
\hline 4 & $11(3.9)$ & $979(6.1)$ & $830(8.0)$ & $1820(6.8)$ \\
\hline \multicolumn{5}{|c|}{ History of alcohol consumption at ART start } \\
\hline No & $212(92.6)$ & $12513(92.5)$ & $6409(75.2)$ & $18869(85.8)$ \\
\hline Yes & $9(3.9)$ & $993(7.5)$ & $2113(24.8)$ & $3117(14.2)$ \\
\hline \multicolumn{5}{|c|}{ History of smoking at ART start } \\
\hline No & $215(96.8)$ & $12147(90.0)$ & $6332(72.9)$ & $18691(83.4)$ \\
\hline Yes & $9(3.9)$ & $737(5.5)$ & $2058(18.6)$ & $2359(10.5)$ \\
\hline \multicolumn{5}{|c|}{ STI in the first 24 months of ART } \\
\hline No & $265(94.6)$ & $14786(91.6)$ & $9805(94.9)$ & $24856(92.9)$ \\
\hline One or more episodes & $15(5.4)$ & $1364(8.4)$ & $527(5.1)$ & $1906(7.1)$ \\
\hline \multicolumn{5}{|c|}{ History of TB at ART start } \\
\hline Yes & $23(8.2)$ & $959(5.9)$ & $803(7.8)$ & $1785(6.7)$ \\
\hline No & $257(91.8)$ & $15191(94.1)$ & $9529(92.2)$ & $24977(93.3)$ \\
\hline
\end{tabular}

Table 3. Proportions of sexually transmitted infections in ART patients in a public hospital in Johannesburg, South Africa

\begin{tabular}{ll}
\hline Sexually transmitted infection & $\boldsymbol{n}(\%)$ \\
\hline Chancroid & $2(0.1)$ \\
Chlamydia & $10(0.5)$ \\
Syphilis & $15(0.8)$ \\
Gonococcal infection & $11(0.6)$ \\
Herpex simplex & $261(13.7)$ \\
Trichomoniasis & $18(0.9)$ \\
Anogenital warts & $310(16.3)$ \\
Unspecified sexually transmitted & $81(4.2)$ \\
Syndromic management & $1198(67.1)$ \\
ART $=$ antiretroviral therapy. &
\end{tabular}

Similarly, the risk of STI diagnosis was higher among patients who were in WHO stages 3 (aHR 1.1; 95\% CI 1.0 - 1.3) or 4 (aHR 1.6; $95 \%$ CI $1.3-1.9$ ) at ART start compared with those in WHO stage 1. In contrast, there was a $20 \%$ increase in the risk of STI diagnosis among obese patients compared with those who were underweight at ART initiation (aHR 1.3; 95\% CI 1.0 - 1.7). However, $>80 \%$ of obese patients who experienced an incident STI had a CD4 count $<200$ cells $/ \mu \mathrm{L}$

\section{Discussion}

We sought to determine the incidence of STIs among HIVpositive adult patients in their first 24 months of HIV treatment in Johannesburg. Overall, 7\% of our sample had at least one STI episode in the first 2 years on ART, at an incidence rate of 4.8/100 py. This 


$\begin{aligned} & \text { Table 4. Drug regimen used to identify incident sexually transmitted infections including vaginal discharge syndrome, male } \\
& \text { urethritis syndrome and lower abdominal pain }\end{aligned}$
\begin{tabular}{llll}
\hline Drug regimen & Female $(\boldsymbol{N}=\mathbf{9 1 1}), \boldsymbol{n}(\%)$ & Male $(\boldsymbol{N}=\mathbf{2 8 7}), \boldsymbol{n}(\%)$ & Total $(\boldsymbol{N}=\mathbf{1} \mathbf{1 9 8}), \boldsymbol{n}(\%)$ \\
\hline Metronidazole + ciprofloxacin & $239(26.2)$ & $154(53.7)$ & $393(32.8)$ \\
Metronidazole + ciprofloxacin + doxycycline & $277(30.4)$ & $52(18.1)$ & $329(27.5)$ \\
Metronidazole + doxycycline & $208(22.5)$ & $23(8.0)$ & $231(19.3)$ \\
Metronidazole + clotrimazole & $53(5.8)$ & 0 & $53(4.4)$ \\
Doxycycline + ciprofloxacin & $21(2.3)$ & $27(9.4)$ & $48(4.0)$ \\
Metronidazole + doxycycline + acyclovir & $38(4.2)$ & $6(2.1)$ & $44(3.7)$ \\
Fluconazole + acyclovir & $28(3.1)$ & $12(4.2)$ & $40(3.3)$ \\
Metronidazole + doxycycline + amoxicillin & $24(2.6)$ & $5(1.7)$ & $29(2.4)$ \\
Other & $23(2.5)$ & $8(2.8)$ & $31(2.6)$
\end{tabular}

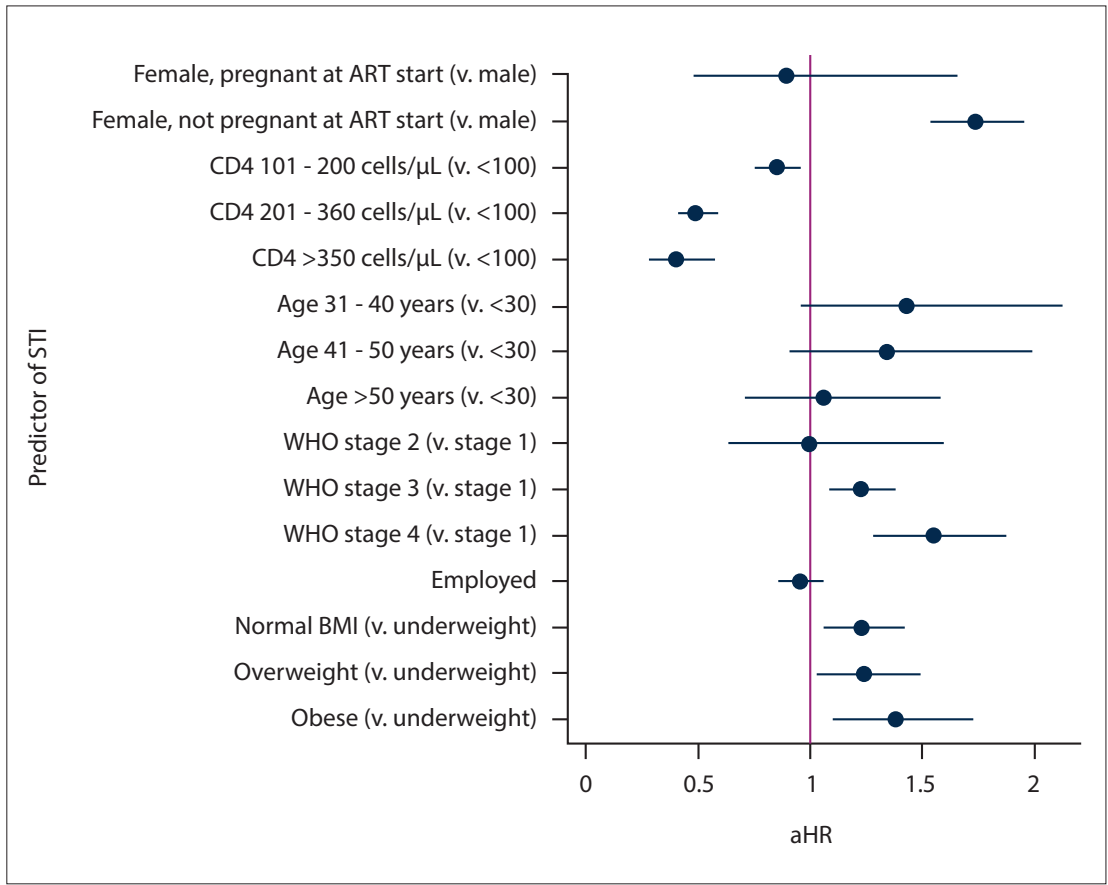

Fig. 1. Adjusted baseline predictors of incident STI among HIV-positive patients receiving ART at the Themba Lethu HIV clinic in Johannesburg, South Africa. (STI = sexually transmitted infection; ART = antiretroviral therapy; $W H O=$ World Health Organization; $B M I=$ body mass index $;$ aHR $=$ adjusted hazard ratio.)

proportion is substantially lower than the $19.9 \%$ estimate from a survey among HIVpositive adults in the Diepsloot community of Johannesburg. ${ }^{[13]}$ However, only $10.2 \%$ of the participants in Diepsloot were receiving ART at the time.

Women who were not pregnant at ART initiation had a higher STI incidence (5.7/100 py) than men, which is much lower than the estimated $20 / 100$ py incidence estimates among HIV-negative female trial participants in SA. ${ }^{[17]}$ Similar to previous studies, our results also indicate that younger women had a higher STI risk than men. ${ }^{[17]}$ The higher risk among nonpregnant women may be associated with their higher healthcare-seeking tendencies compared with men. ${ }^{[18]}$
Predictors of STIs included a low CD4 count, advanced WHO stage and high BMI at ART initiation. While we did not report on health status indicators at the time of the STI episodes, our findings are consistent with other studies showing that immunocompromised individuals are generally more vulnerable to STIs. ${ }^{[7]}$ Patients with weak immune systems (as indicated by a low CD4 count) but in apparent good health (high BMI) seem to be at high risk. However, there could also be a potential diagnostic bias in a population that is already in HIV care. Patients with more severe HIV disease may have high healthcare-seeking behaviour, thereby increasing the likelihood of STI diagnosis. Also, patients with a low CD4 count may be subject to more intensive investigations, including laboratory tests for STIs and other pathogens.

Nevertheless, STIs in this population primarily indicate high-risk sexual activity. Viral load data were minimal among patients with STI episodes. However, the majority of those with viral load data were suppressed. While HIV treatment reduces infectiousness by controlling viral load, this is only the case among ART-adherent and virally suppressed patients. The risk for onward HIV transmission remains a reality among patients with transient viral suppression, particularly during an STI episode. ${ }^{[19,20]}$ Patients should therefore be encouraged to use barrier methods and to seek healthcare as soon as STI symptoms manifest.

\section{Study limitations}

The data for this analysis were drawn from a single HIV clinic in Johannesburg that may not be representative of other settings in SA. Furthermore, the reliance on diagnosis notes and prescription data does not take into account asymptomatic patients who did not present for care and introduces the potential for care-seeking and diagnostic bias. Also, the sensitivity of this indirect prescription-based STI identification method must be assessed against direct sample testing to establish the margin of error to expect in monitoring efforts under the syndromic management policy in SA. Nevertheless, further research is needed to explore methods for improving data for STI monitoring among patients in HIV care in SA.

\section{Conclusions}

STIs are common in HIV-infected patients who are receiving ART. While both ART and the syndromic management of STIs are high-impact interventions for controlling the spread of HIV, closer monitoring of STI occurrences is warranted, particularly among immunologically vulnerable patients. 


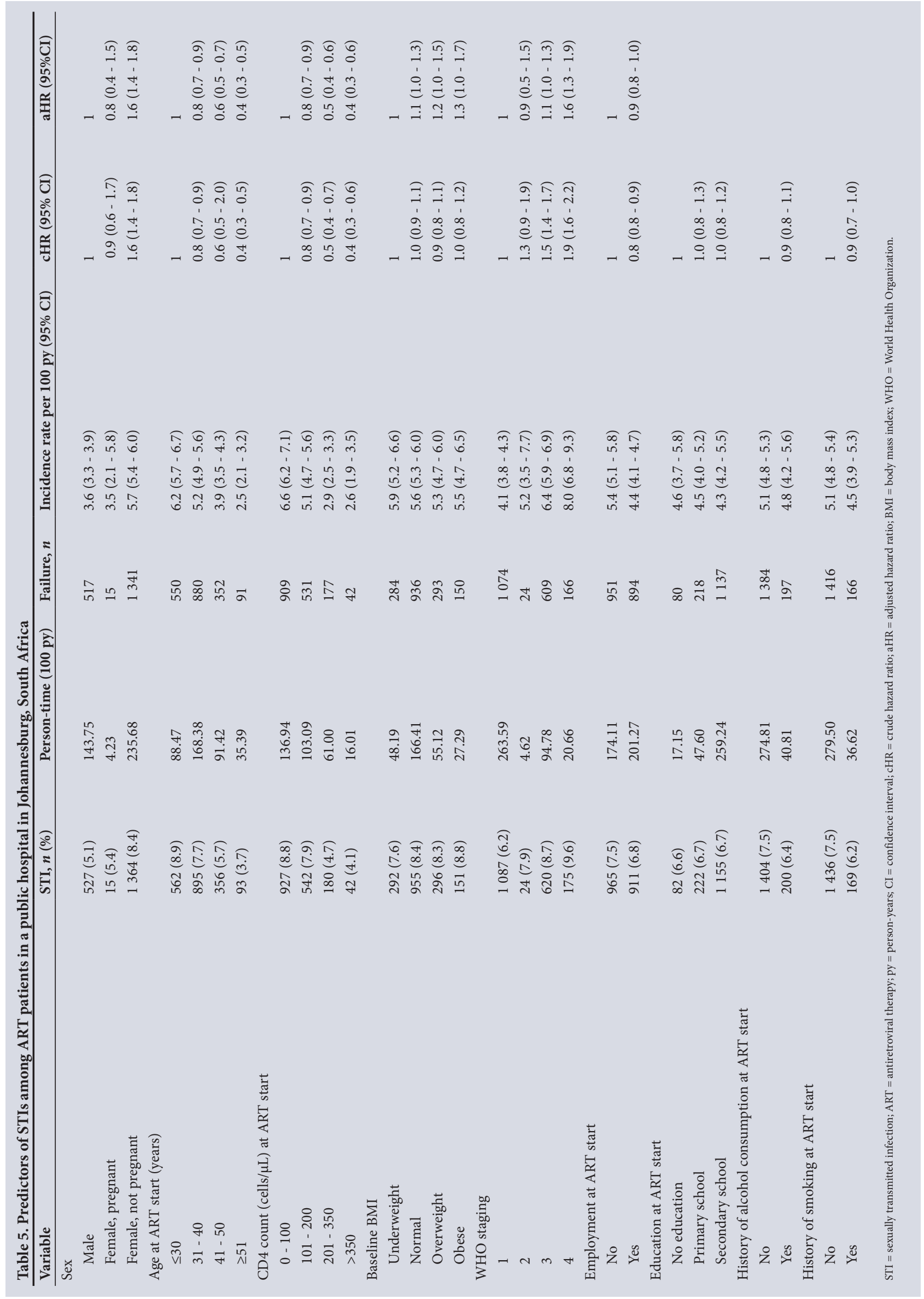




\section{Declaration. None}

Acknowledgements. The authors gratefully acknowledge the directors and staff of the Themba Lethu HIV clinic as well as Right to Care, the non-governmental organisation supporting the study site through a partnership of the SA national and Gauteng provincial departments of health with the United States Agency for International Development (USAID). Most of all we thank the patients attending the Themba Lethu clinic for their continued trust in the treatment provided.

Author contributions. PM, CN, SK, MM and MPF contributed equally to the work. DO, PM, CN and MPF conceptualised the analysis. PM and SK analysed the data and drafted the manuscript. KS and MM assisted in the analysis of data and contributed to the interpretation of the results as well as the manuscript preparation.

Funding. This study has been made possible by the generous support of the American people and the President's Emergency Plan for AIDS Relief (PEPFAR) through USAID under the terms of Cooperative Agreements AID-674-A-12-00029 and 72067419CA00004 to the Health Economics and Epidemiology Research Office, Cooperative Agreement 674-A-0009-00018-00 to Boston University and Cooperative Agreement 674-A-1200020 to Right to Care. The contents are the responsibility of the authors and do not necessarily reflect the views of PEPFAR, USAID or the US government. The funders had no role in the study design, collection, analysis and interpretation of the data, manuscript preparation, or the decision to publish.

\section{Conflicts of interest. None.}

1. Mayaud P, Mabey D. Approaches to the control of sexually transmitted infections in developing countries: Old problems and modern challenges. Sex Transm Infect 2004;80(3):174-182. https://doi. org/10.1136/sti.2002.00410

2. Kularatne RS, Niit R, Rowley J, et al. Adult gonorrhoea, chlamydia and syphilis prevalence, incidence, treatment and syndromic case reporting in South Africa: Estimates using the Spectrum-STI model, 1990 - 2017. PLoS ONE 2018;13(10):e0205863. https://doi.org/10.1371/journal.pone.0205863

3. National Department of Health, South Africa. The 2015 National Antenatal Sentinel HIV \& Syphilis 03-29-10-42-47/2015-04-30-08-18-10/2015-04-30-08-21-56?download=2584:2015-nationalantenatal-hiv-prevalence-survey-final-23oct17 (accessed 18 November 2020).
4. Basera TJ, Takuva S, Muloongo K, Tshuma N, Nyasulu PS. Prevalence and risk factors for self-reported sexually transmitted infections among adults in the Diepsloot informal settlement, Johannesburg, South Africa. J AIDS Clin Res 2016;7:539. https://doi.org/10.4172/2155-6113.1000539

5. Johnson LF, Dorrington RE, Moolla H. Progress towards the 2020 targets for HIV diagnosis and Johnson LF, Dorrington RE, Moolla H. Progress towards the 2020 targets for HIV diagnosis and
antiretroviral treatment in South Africa. South Afr J HIV Med 2017;18(1):1-8. https://doi.org/10.4102/ antiretroviral treatm
sajhivmed.v18i1.694

6. Newman L, Rowley J, Vander Hoorn S, et al. Global estimates of the prevalence and incidence of four curable sexually transmitted infections in 2012 based on systematic review and global reporting. PLoS ONE 2015;10(12):e0143304. https://doi.org/10.1371/journal.pone.0143304

7. Haggerty CL, Gottlieb SL, Taylor BD, Low N, Xu F, Ness RB. Risk of sequelae after Chlamydia trachomatis genital infection in women. J Infect Dis 2010;201(Suppl 2):S134-S155. https://doi org $/ 10.1086 / 652395$

8. Johnson LF, Chiu C, Myer L, et al. Prospects for HIV control in South Africa: A model-based analysis. Glob Health Action 2016;9:30314. https://doi.org/10.3402/gha.v9.30314

9. Johnson LF, Dorrington RE, Bradshaw D, Coetzee DJ. The role of sexually transmitted infections in the evolution of the South African HIV epidemic. Trop Med Int Health 2012;17(2):161-168. https://doi. org/10.1111/j.1365-3156.2011.02906.x

10. Sexton J, Garnett G, Rottingen JA. Meta-analysis and metaregression in interpreting study variability in the impact of sexually transmitted diseases on susceptibility to HIV infection. Sex Transm Dis in the impact of sexually transmitted diseases on susceptibility to HIV infection. Sex Transm Dis

1. Bosu WK. Syndromic management of sexually transmitted diseases: Is it rational or scientific? Trop Med Int Health 2002;4(2):114-119. https://doi.org/10.1046/j.1365-3156.1999.00360.x

2. National Institute for Communicable Diseases. GERMS South Africa: Annual Report 2017 Johannesburg: NICD, 2017. https://www.nicd.ac.za/wp-content/uploads/2018/08/GERMS-SA-AR2017-final.pdf (accessed 18 November 2020).

13. Kaida A, Dietrich JJ, Laher F, et al. A high burden of asymptomatic genital tract infections undermines the syndromic management approach among adolescents and young adults in South Africa: Implications for HIV prevention efforts. BMC Infect Dis 2018;18(1):499. https://doi.org/10.1186 s12879-018-3380-6

14. World Health Organization. Report on global sexually transmitted infection surveillance 2018 . Geneva: WHO, 2018. https://www.who.int/reproductivehealth/publications/stis-surveillance-2018/ en/ (accessed 18 November 2020).

15. National Department of Health, South Africa. National consolidated guidelines for the prevention of mother-to-child transmission of HIV (PMTCT) and the management of HIV in children, adolescents and adults. Pretoria: NDoH, 2015. https://sahivsoc.org/Files/ART\%20Guidelines\%2015052015.pdf and adults. Pretoria: NDoH,

16. National Department of Health, South Africa. Sexually transmitted infections management guidelines, 2015. Pretoria: NDoH, 2015. https://health-e.org.za/2015/06/25/guidelines-sexually-transmittedinfection-management-guidelines-2015/ (accessed 18 November 2020).

17. Naidoo S, Wand H, Abbai NS, Ramjee G. High prevalence and incidence of sexually transmitted infections among women living in Kwazulu-Natal, South Africa. AIDS Res Ther 2014;11:31. https:// doi.org/10.1186/1742-6405-11-31

8. Backonja U, Royer HR, Lauver DR. Young women's reasons to seek sexually transmitted infection screening. Public Health Nurs 2014;31(5):395-404. https://doi.org/10.1111/phn.12125

19. Cohen MS, Gay CL. Treatment to prevent transmission of HIV-1. Clin Infect Dis 2010;50(0 3):S85-S95. https://doi.org/10.1086\%2F 651478

20. Kalichman SC, Pellowski J, Turner C. Prevalence of sexually transmitted co-infections in people living with HIV/AIDS: Systematic review with implications for using HIV treatments for prevention. Sex Transm Infect 2011;87(3):183-190. https://doi.org/10.1136\%2Fsti.2010.047514 